Article

\title{
Expansion and Functional Diversification of SKP1-Like Genes in Wheat (Triticum aestivum L.)
}

\author{
Imen HajSalah El Beji, Said Mouzeyar, Mohammed-Fouad Bouzidi and Jane Roche *(iD \\ UMR INRA/UCA 1095 GDEC, Université Clermont Auvergne, Campus Universitaire des Cézeaux, 1 \\ Impasse Amélie Murat, TSA 60026 AUBIERE, France \\ * Correspondence: Jane.roche@uca.fr; Tel.: +33-(47)-3407939
}

Received: 6 May 2019; Accepted: 2 July 2019; Published: 4 July 2019

\begin{abstract}
The ubiquitin proteasome $26 \mathrm{~S}$ system (UPS), involving monomeric and multimeric E3 ligases is one of the most important signaling pathways in many organisms, including plants. The SCF (SKP1/Cullin/F-box) multimeric complex is particularly involved in response to development and stress signaling. The SKP1 protein (S-phase kinase-associated protein 1) is the core subunit of this complex. In this work, we firstly identified 92 and 87 non-redundant Triticum aestivum SKP1-like (TaSKP) genes that were retrieved from the latest release of the wheat genome database (International Wheat Genome Sequencing Consortium (IWGSC) RefSeq v1.0) and the genome annotation of the TGAC v1 respectively. We then investigated the structure, phylogeny, duplication events and expression patterns of the SKP1-like gene family in various tissues and environmental conditions using a wheat expression platform containing public data. TaSKP1-like genes were expressed differentially in response to stress conditions, displaying large genomic variations or short insertions/deletions which suggests functional specialization within TaSKP1-like genes. Finally, interactions between selected wheat FBX (F-box) proteins and putative ancestral TaSKP1-like proteins were tested using the yeast two-hybrid ( $\mathrm{Y} 2 \mathrm{H})$ system to examine the molecular interactions. These observations suggested that six Ta-SKP1 genes are likely to be ancestral genes, having similar functions as ASK1 and ASK2 in Arabidopsis, OSK1 and OSK20 in rice and PpSKP1 and PpSKP2 in Physcomitrella patens.
\end{abstract}

Keywords: SKP1; Triticum aestivum; structural and evolution analysis; $\mathrm{Y} 2 \mathrm{H}$

\section{Introduction}

Selective degradation of proteins plays a key role in diverse aspects of eukaryotic mechanisms such as responses to physiological development (flowering, cell cycle, etc.) or in responses to diverse environmental stresses [1-6] by degrading target proteins that play a role in the activation or repression of downstream genes. The substrate selectivity is insured by E3 ubiquitin ligases which could be monoor multi-subunit complexes. Among the multi-subunit E3 ubiquitin ligases complexes, the SKP1/Cullin 1/F-box protein (SCF) family is particularly well characterized [7,8]. Each SCF complex is composed of four protein components: the Cul1-Rbx1 (Cullin 1/ RING-box protein 1) catalytic core bound to a variable F-box protein (FBX)-Skp1 substrate recognition module $[9,10]$.

SKP1 is a small protein of approximately 160 amino acids and functions as a core component connecting CUL1 and an F-box protein. SKP1 plays crucial roles in cell-cycle progression, in hormone and light signaling [11-16] as well as in vegetative and flower development [17-21].

Only one SKP1 gene has been described in protists, algae and fungi [22]. In contrast, plants have multiple SKP1 genes that appeared as a consequence of duplication events leading to diverse functions [22-26]. SKP1-like gene evolution in several plant species was investigated by Kong et al. [22] who suggested that all the SKP1 genes found in these species were originated from one ancestral gene called ASK1 in Arabidopsis and OSK1 in rice, which may have similar functions [22,26]. Further, SKP1 
genes were described as belonging to three different classes by Kong et al. [26]. Type Ia corresponds to $S K P$ genes containing one intron, Type Ib corresponds to intronless SKP genes, and Type II corresponds to $S K P$ genes containing more than one intron. Furthermore, Kong et al. [26], then Kahloul et al. [23], suggested that ancestral SKP1 genes typically contain one intron whose position is conserved within the genomic sequence.

To gain further insight into the characterization of wheat SKP1-like genes, we carried out a comprehensive analysis of this gene family. We investigated the structure, phylogeny, duplication events and expression patterns of the SKP1-like gene family in various tissues and environmental conditions using a wheat expression platform [27]. All Triticum aestivum SKP1-like (TaSKP1) genes were retrieved using data from the latest release of the wheat genome database (International Wheat Genome Sequencing Consortium (IWGSC) RefSeq v1.0, https://www.wheatgenome.org/) and TGAC v1 [28] annotated genome reference. Analysis of the structure, the expansion and the expression of this family of genes was conducted. In addition, we also analyzed the homoeology relationships between the subgenomes A, B and D that constitute the hexaploid wheat genome. Because SKP1 and FBX proteins interact to form the SCF complex, the yeast two-hybrid (Y2H) system was used to test for the physical interactions between certain wheat TaSKP1 proteins, considered to be ancestral proteins, and three wheat FBX already cloned in the laboratory (TaFBX proteins).

\section{Results}

\subsection{Genome-Wide Identification of TaSKP1-Like Genes}

HMMER2-BLASTP-InterProScan searches of databases containing the latest version of the annotated wheat genome database (International Wheat Genome Sequencing Consortium (IWGSC RefSeq v1.0)) and in TGAC v1 [28] identified 92 (which we designated TaSKP1_1 through TaSKP1_92) and 87 non-redundant Triticum aestivum SKP1-like genes, respectively, with conserved Skp1 and Skp1_POZ (POxvirus and Zinc finger) domains.

Subsequently, we established the correspondence (one-to-one) between the two annotations for the TaSKP1-like genes (Table S1).

\subsection{Chromosomal Location of TaSKP1-Like Gene}

By using their coordinates on the pseudomolecule, we found that the 92 sequences were located on three subgenomes A, B and D (Table 1). It appears that each chromosome of each subgenome contains at least one TaSKP1-like gene (Figure 1). However, the distribution between chromosomes was not even. Only chromosomes Chr1A and Chr1B each contained a single copy, whereas the other chromosomes contained several TaSKP1-like genes. For example, the 3D, 3B and 5D chromosomes contained 8, 9 and 10 copies, respectively. In addition, when a chromosome contains several copies, some of these copies are grouped into clusters and sometimes in a very narrow region such as the TaSKP1_49, TaSKP1_50,TaSKP1_51 and TaSKP1_52 genes, which were clustered within $61 \mathrm{~kb}$ on the 2D chromosome or the TaSKP1_57 and TaSKP1_58 genes which were clustered within $72 \mathrm{~kb}$ on the 3B chromosome. In addition, TaSKP1-like genes belonging to the same family tended to be clustered within the same chromosomal location. For instance, TaSKP1_47 through TaSKP1_53, which are intronless genes, were clustered in the distal part of the 2D chromosome. Similarly, TaSKP1_23, TaSKP1_24,TaSKP1_25 and TaSKP1_26, which contain one intron in a conserved position, were located within the same region on the $7 \mathrm{~B}$ chromosome (Figure 1). 


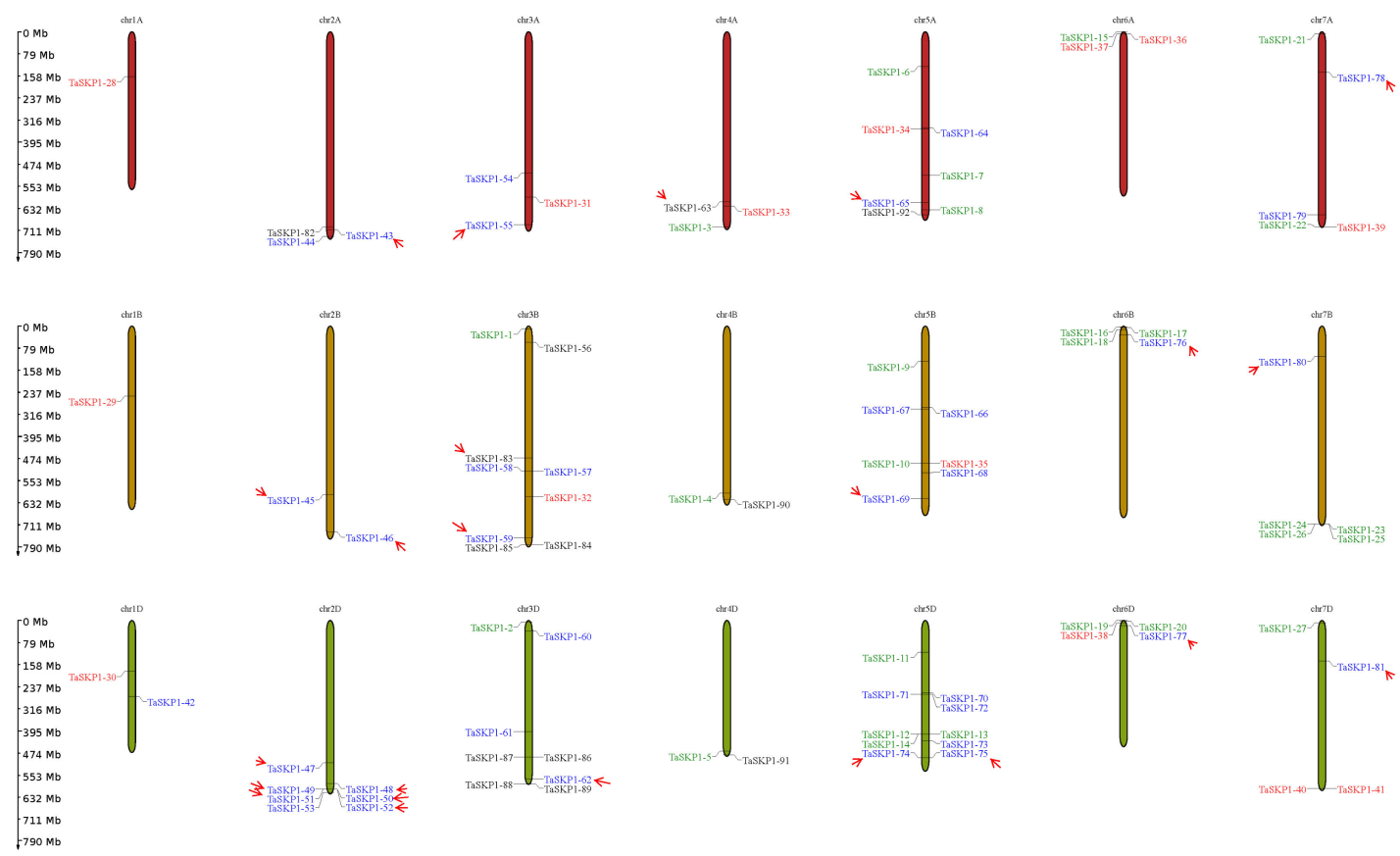

Figure 1. Chromosomal repartition of the 92 TaSKP1-like genes on the 21 chromosomes of the wheat genome (A, B and D subgenomes). Colors indicate genes belonging to the same structural class (see Table S1). Red arrows indicate the genes considered for duplication analysis (see Table S4).

Table 1. The distribution of 92 TaSKP1-like genes (International Wheat Genome Sequencing Consortium (IWGSC) RefSeq) in T. aestivum 21 chromosomes.

\begin{tabular}{cccc}
\hline Chromosome & A Subgenome & B Subgenome & D Subgenome \\
\hline 1 & 1 & 1 & 2 \\
2 & 3 & 2 & 7 \\
3 & 3 & 9 & 8 \\
4 & 3 & 2 & 2 \\
5 & 7 & 7 & 10 \\
6 & 3 & 4 & 4 \\
7 & 5 & 5 & 4 \\
\hline
\end{tabular}

\subsection{Phylogeny of the TaSKP1-Like Genes}

As shown in Figure 2, a phylogenetic tree of wheat TaSKP1-like genes was inferred using the maximum likelihood method. Several clades contained genes belonging to the same class determined by Kong et al. [26] and Kahloul et al. [23]. In particular, it can be noticed that there was a large clade comprising 23 TaSKP1-like genes, among which 22 were intronless (class $\mathrm{Ib}$ genes) and only one gene (TaSKP1_83) contained several introns (class II). Moreover, the length of the branches in this clade reflects a high sequence similarity between its members. Similarly, one clade included most of the genes with more than one intron (TaSKP1_86, TaSKP1_82, TaSKP1_87, TaSKP1_84, TaSKP1_88, TaSKP1_85 and TaSKP1_89)(class II).

The grouping of some sequences with the same structure on the phylogenetic tree as well as the clustering of the same genes on the wheat pseudomolecule suggest that they could be the result of duplication phenomena. 


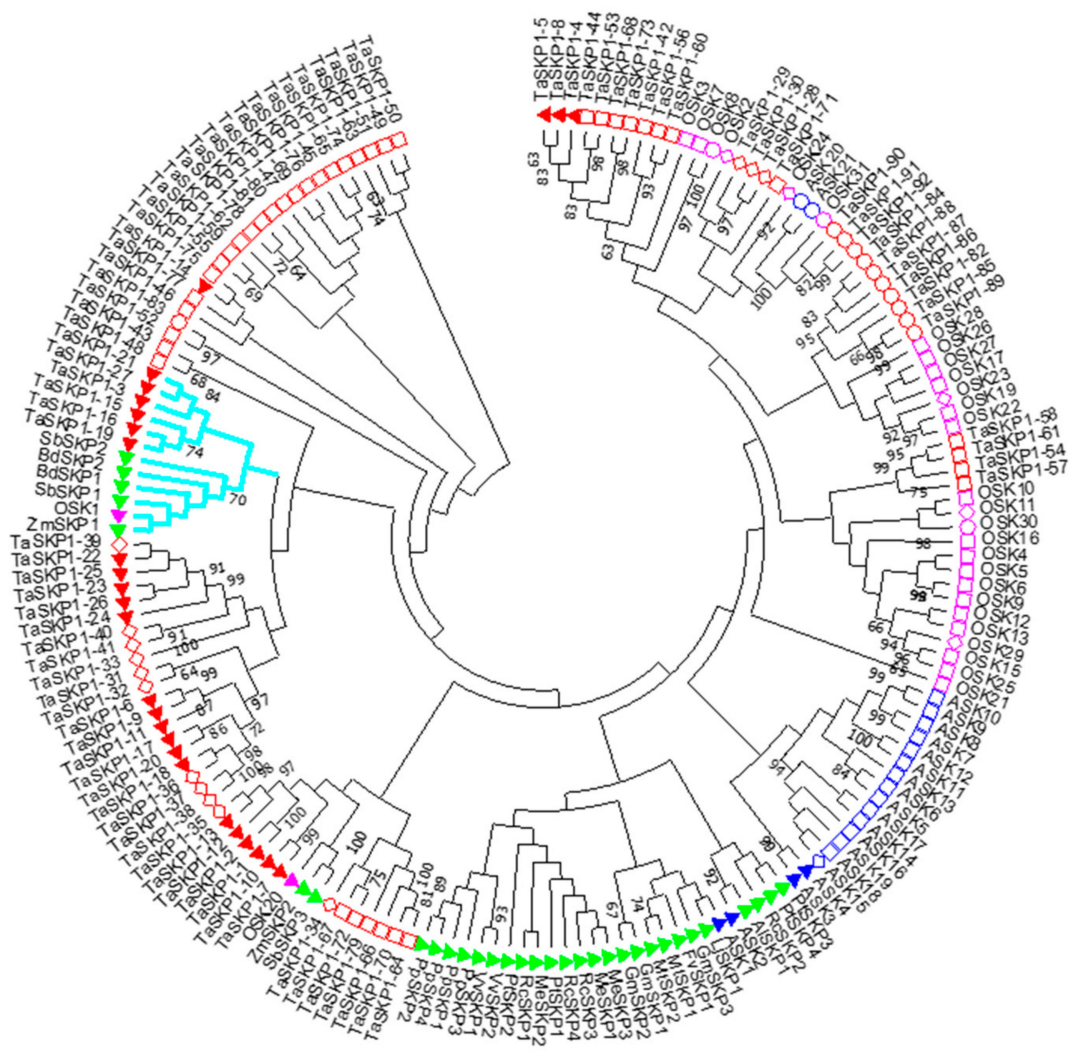

Figure 2. Phylogenetic tree of SKP1-like genes in wheat based on the alignment of the amino acid sequences of 174 SKP-like genes from moss, monocot and eudicot species comprising $92 \mathrm{~T}$. aestivum TaSKP1-like genes (TaSKP1_1 through TaSKP1_92 in red), 4 P. patens genes denoted PpSKP (PpSKP1 through PpSKP4 in green), 21 Arabidopsis thaliana ASK genes (ASK1 through ASK21 in blue), 29 Oryza sativa genes (OSK1 through OSK31 in purple; two of which are no longer included in this denomination). A set of 28 additional SKP1-like genes from various plant species with a single position-conserved intron was included (ZmSKP from Zea mays, SbSKP from Sorghum. bicolor, MtSKP from Medicago truncatula, RcSKP from Ricinus communis, PtSKP from Populus trichocarpa, GmSKP from Glycin max, VvSKP from Vitis vinefera, BdSKP from Brachypodium distachyon, LjSKP from Lotus japonicus, AlSKP from Arabidopsis lyrata, MeSKP from Medicago esculenta). The clade colored in blue contains the genes with a single intron in a conserved position.

\subsection{Homoeologous Group Identification, Structure and Expression Profiles of TaSKP1-Like Genes}

To identify homoeologous sequences, CD-HIT suite was used with sequence identity cut-off set at $90 \%$, meaning that sequences with identity percent above 90 are clustered together. So, 42 clusters were identified and numbered from cluster \#0 to cluster \#41 (Table S2). The cluster \#0 was the largest one and contained 18 sequences. Twenty-three clusters (clusters \#19 through \#41) each contained only one sequence. To identify homoeologous sequences, clusters containing two or three members that were originating from the same chromosome in subgenomes A, B and D were considered as homoeologous groups. For example, cluster \#3 contained TaSKP1_54, TaSKP1_58 and TaSKP1_61, indicating that it is likely a triplet of homoeologous genes in the third chromosome. Similarly, cluster \#13 contained TaSKP1_44 and TaSKP_53 corresponding to a pair of homoeologous genes. In contrast, cluster \#11 and cluster \#12 contained pairs of highly similar sequences but were located in non-homoeologous chromosomes. In addition, cluster \#0 and cluster \#2 contained more than three members, among which some were considered as "probable homoeologs" because they were located in homoeologous chromosomes (Table S2). 
In summary, eight triplets and 11 pairs yielding a total of 46 members were identified as homoeologous groups, while the 46 remaining sequences were considered as "singlets" without any obvious homoeologous counterpart.

Considering the homoeolog structure, all the homoeologs displayed the same exon-intron organization, except cluster \#7 and cluster \#0. In cluster \#7, TaSKP1_34 contained one intron while TaSKP1_67 and TaSKP1_72 did not, suggesting that the A copy could have acquired an intron after polyploidisation. Simarly, TaSKP1_76 contained one intron while its probable homoeolog TaSKP1_77 was intronless.

Then, homoeolog triplets' and pairs' expression profiles were followed in different seed tissues (grains, spikes, leaves and roots) [29]. Independently to the homoeologous groups identified, TaSKP1-like genes expression patterns appear to be diverse (Table S3). In the case of triplets, five of them exhibited similar expression between the homoeologs, while three triplets experienced partitionning of expression between the homoeologs. Indeed, one or two homoeologs were expressed differently when compared to the other homoeologs. For example, TaSKP1_7, TaSKP1_10 and TaSKP1_14 formed a triplet within which, the $\mathrm{A}$ and $\mathrm{B}$ homoeologs were more expressed thant the D homoeolog. The same situation was observed concerning pairs of homoeologs. For example, while TaSKP1_4 (B subgenome) and TaSKP_5 (D subgenome) exhibited almost identical expression profiles, the pair formed by TaSKP1_56 (B subgenome) and TaSKP1_60 (D subgenome) exhibited a very contrasting expression with no expression detected for the D homoeolog.

\subsection{Duplications}

In order to investigate the hypothesis of duplication, approximative lengths of $100 \mathrm{~kb}$ on either side (i.e., regions of about $200 \mathrm{~kb}$ ), of each TaSKP1-like gene belonging to the same clade was selected for further investigation. As an example, a clade containing 23 intronless genes was selected as indicated in yellow in Figure S1 and Table S4. In the cases of TaSKP1_49, TaSKP1_50, TaSKP1_51 and TaSKP1_52, the genes were very close (separated by less than $100 \mathrm{~kb}$ ), hence a region of $261.018 \mathrm{~kb}$ containing these genes was used. Similarly, a region of $255.143 \mathrm{~kb}$ which contained TaSKP1_74 and TaSKP1_75 was used. Using the Yass tool, several highly conserved regions were identified between these two regions, which are likely the result of duplication phenomena. This is illustrated in Figure 3, which represents duplicated fragments that can be identified. To clarify the nature and extent of duplication, we identified all duplicated fragments between loci that were at least $3 \mathrm{~kb}$ in size and had 90\% identity (Table S4). Thus, in total, we identified 122 non-overlapping duplicate fragments whose lengths ranged from 3.098 to $12.619 \mathrm{~Kb}$ (Figure $4 \mathrm{a}$ and Table S4). Figure $4 \mathrm{~b}$ presents duplication relationships between members of these clades. The largest number of duplicate fragments between two non-homoeologous loci involved the TaSKP1_49-TaSKP1_50-TaSKP1_51-TaSKP1_52 locus on the 2D chromosome which shared 16 fragments with other loci, and the TaSKP1_74-TaSKP1_75 locus on the 5D chromosome which shared 29 fragments with other loci. The majority of duplications were around $4 \mathrm{~kb}$ (median duplication size $=5.960 \mathrm{~kb}$, mean duplication size $=6.208 \mathrm{~Kb}$ ). The largest duplicate fragment was 13,675 $\mathrm{kb}$ in length between the loci containing TaSKP1_74 and TaSKP1_75 on the 5D chromosome and the locus containing TaSKP1_47 on the 2D chromosome. 


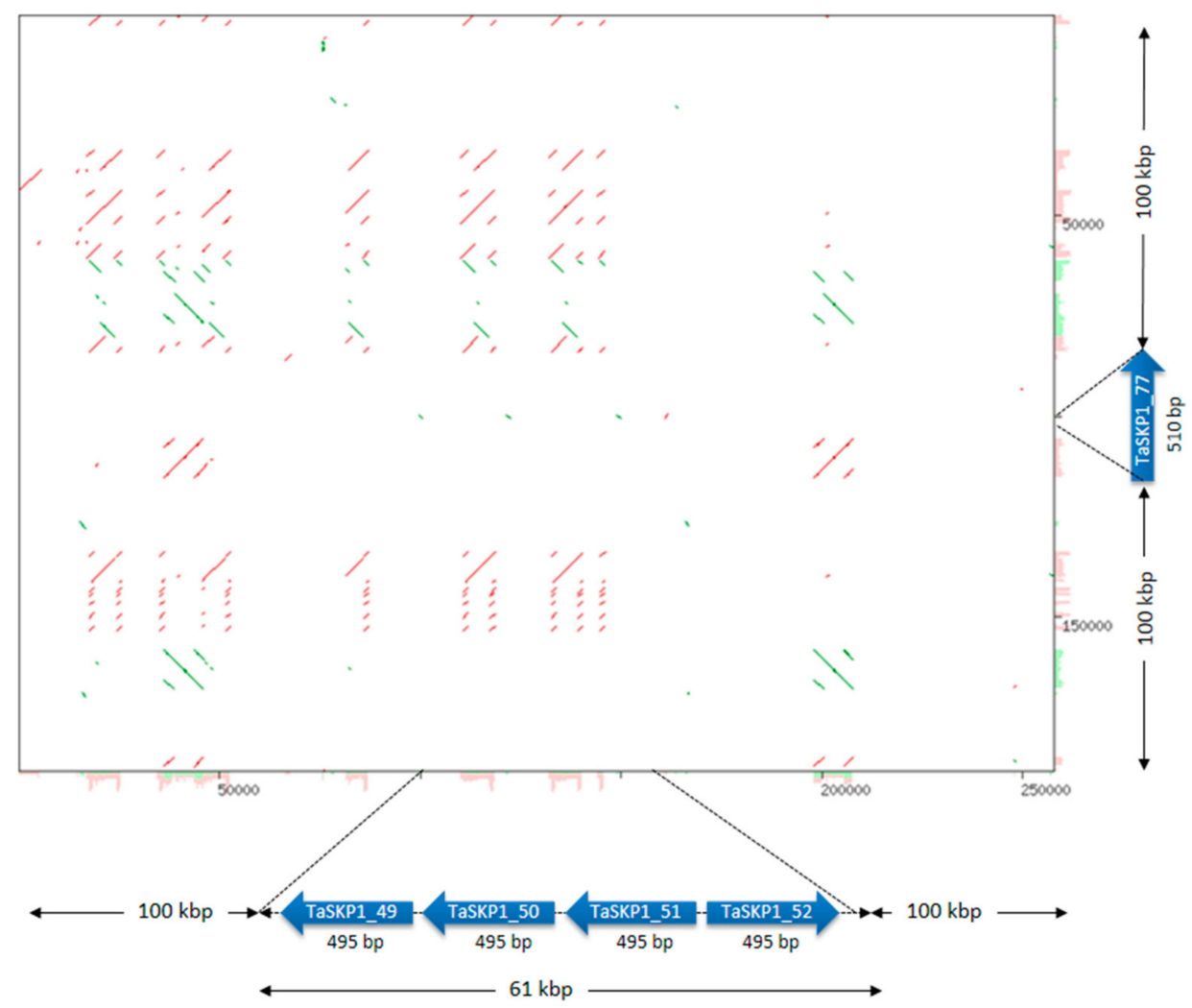

Figure 3. Dotplot showing an example of duplications between two genomic regions containing TaSKP1_49,TaSKP1_50,TaSKP1_51 and TaSKP1_52 genes and TaSKP1_74 and TaSKP1_75 genes. The red dots correspond to the sense duplications and the green dots to the antisense duplications.
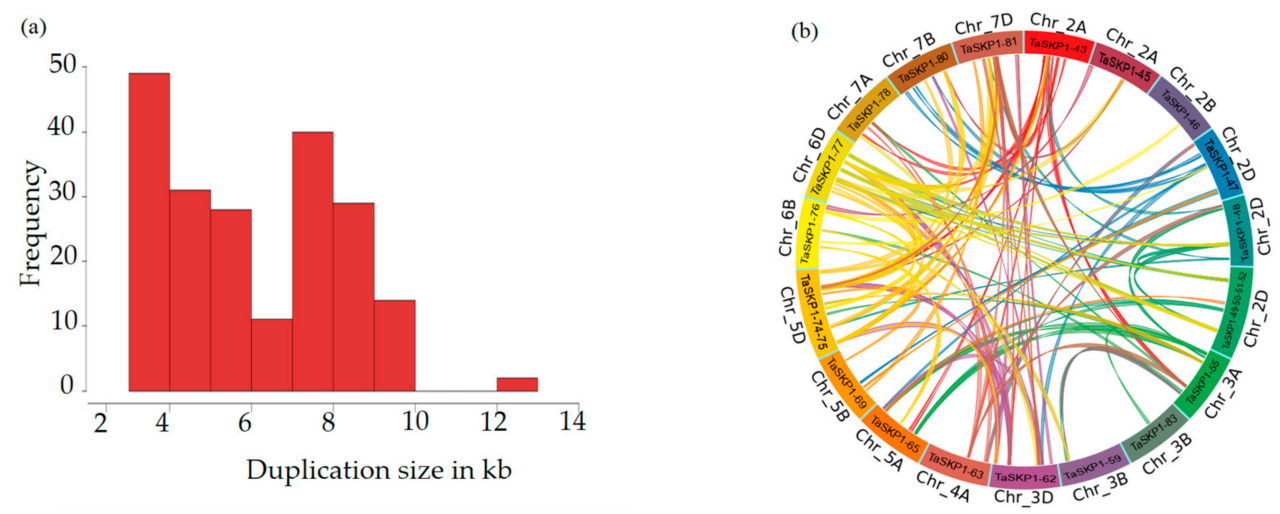

Figure 4. (a) Frequency and size of duplicated regions on the wheat genome and (b) duplicate events in the relevant phylogenetic clades. Each connection represents a duplicated sequence with a minimum size of $3 \mathrm{~Kb}$ and at least $90 \%$ nucleic identity.

\subsection{Structural and Phylogenic Analysis of TaSKP1-Like Genes}

Nucleotide and protein alignments indicated that TaSKP1_1 to TaSKP1_27 genes contained a unique intron in a conserved position, meaning that the second exon is $174 \mathrm{bp}$ long while the first exon and the intron could be variable in length. These genes were all classified as Type Ia $S K P$ genes under Kong et al.'s system. TaSKP1_28 to TaSKP1_41 genes contained one non-conserved intron and were all classified as Type Ia. TaSKP1_42 to TaSKP1_81 intronless genes were all classified as Type Ib. TaSKP1_82 to TaSKP1_92 genes contained more than one intron and were all classified as Type II.

Phylogenetic analysis of the 174 SKP1-like genes from the moss, monocot and eudicot species (see Material and Methods) indicated that TaSKP1_3, TaSKP1_15, TaSKP1_16, TaSKP1_19, TaSKP1_21, 
TaSKP1_27 and six other monocot SKP1-like genes (OSK1, ZmSKP1, SbSKP1, SbSKP2, BdSKP1 and $B d S K P 2$ ) that contained a single position-conserved intron clustered together (Figure 2). In contrast, the remaining genes clustered in lineage-specific clades which suggests that they formed after the split between wheat and the other species. Interestingly, while the majority of wheat TaSKP1-like genes clustered together, some of them were found in clades containing genes originating from other monocots. For instance, TaSKP1_3, TaSKP1_15, TaSKP1_16, TaSKP1_19, TaSKP1_21 and Ta SKP1_27 were found in a clade containing ZmSKP1 from maize, OSK1 from rice, BdSKP1 and BdSKP2 from Brachypodium and SbSKP2 from sorghum. Indeed, all these genes contain a single intron in a conserved position. A second clade also contained mainly TaSKP1-like genes with single position-conserved intron as well as OSK20 (TaSKP1_7, TaSKP1_10, TaSKP1_1, TaSKP1_2, TaSKP1_12 and TaSKP1_13) (Figure 2).

Kong et al. [22] suggested that ancestral genes such as ASK1 and OSK1 are essential and as such, they evolve slowly and display wide and high expression levels. On the other hand, moderately and rapidly evolving members are more diverse in their genomic structure and are generally less expressed, suggesting that they may have lost their original function(s) and/or acquired new function(s). To test this hypothesis, we further explored the level of expression of TaSKP1-like genes in different tissues (grains, spikes, leaves and roots) during wheat development.

\subsection{Expression Profiles of the TaSKP1-Like Genes during Wheat Development}

To study how TaSKP1-like genes behave during development and in response to stimuli, we selected two RNAseq datasets from the www.wheat-expression.com platform (see Materials and Methods). After filtering the data to eliminate the genes that had a too low level of expression and the ones corresponding to "non-expressed" genes, 78 TaSKP1-like sequences were retained for further analysis. Comparison of normalized levels of expression during different stages of wheat development showed that TaSKP1-like genes exhibit variable behavior (Figure 5, Table S5). One group of TaSKP1-like genes had a constitutive expression in the four analyzed tissues (grains, spikes, leaves and roots), with medium to high expression. These genes are TaSKP1_3, TaSKP1_7, TaSKP1_10, TaSKP1_15, TaSKP1_16, TaSKP1_19, TaSKP1_21, TaSKP1_27, TaSKP1_28, TaSKP1_29, TaSKP1_30, TaSKP1_54, TaSKP1_57, TaSKP1_63, TaSKP1_82, TaSKP1_90,TaSKP1_91 and TaSKP1_92. However, among these genes, some had a significantly higher level of expression than others, regardless of the tissue, such as TaSKP1_15, TaSKP1_16 and TaSKP1_19 genes (average expression level of $11.96 \pm 0.18$ ), followed by TaSKP1_3, TaSKP1_21, TaSKP1_27, TaSKP1_82, TaSKP1_90, TaSKP1_91 and TaSKP1_92 genes (average expression level of $8.49 \pm 0.52$ ).

In addition, 11 other TaSKP1-like genes displayed a high expression level only in grains whereas they were weakly expressed in the other tissues (spike, roots and leaves) and TaSKP1_9 displaying the highest expression level in grains. 
Mean_Normalized_Expression

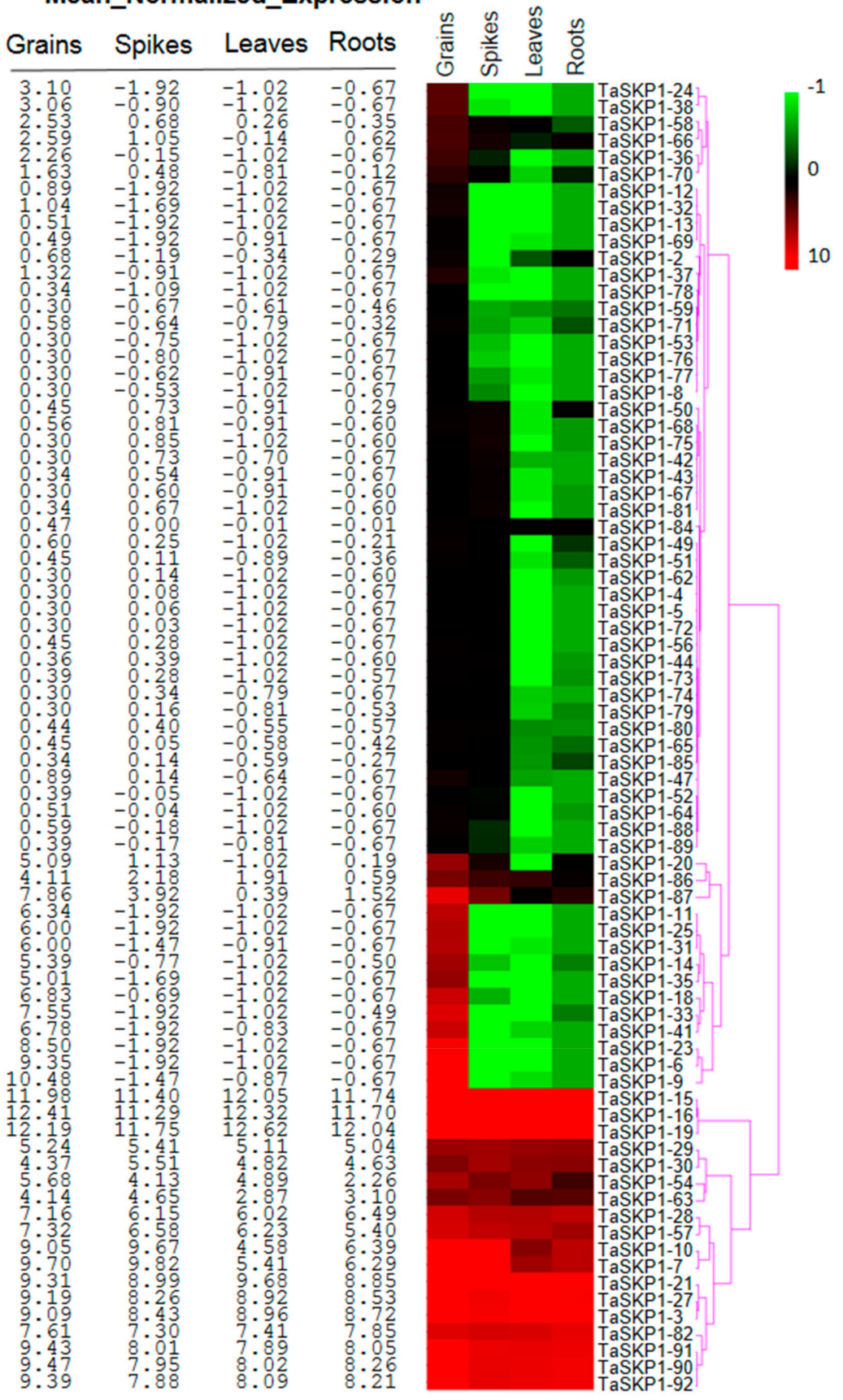

Figure 5. Heat map of the 92 TaSKP1-like genes' expression profiles in wheat grains, spikes, leaves and roots. Hierarchical clustering was performed for the transcript abundance from all tissues. The values indicate the expression level of each gene normalized using the TMM and Voom methods [30,31] after removing non-expressed genes. Highly expressed genes are highlighted in red, weakly expressed genes are highlighted in green.

\subsection{Expression Profiles of the TaSKP1-like Genes in Response to Different Abiotic Stresses in Leaves}

To assess the effects of abiotic stresses on the TaSKP1-like gene expression pattern, the data from Liu et al. [32], which include high temperature, drought and a combination of the two stresses, were extracted to identify differentially expressed genes. Using LIMMA analysis [30], we identified 16 TaSKP1-like genes differentially expressed in at least one treatment (Figure 6a), some were activated under stress while others were repressed. In particular, TaSKP1_64 and TaSKP1_65 were found to 
be up-regulated by heat or a combination of drought and heat one hour after treatment (Figure 6b). This result suggests that there is some degree of functional specialization within the members of this family. When analyzing the correlation between expressions of these genes, it appeared that some of them had strongly positively correlated expression profiles (Pearson coefficient (Pc) close to 1). For example, this was the case for TaSKP_10 and TaSKP_14 $(\mathrm{Pc}=0.98)$ or TaSKP_72 and TaSKP_74 genes $(\mathrm{Pc}=0.99)$. On the other hand, other genes had a weak expression profile, such as TaSKP_71 and TaSKP_72 $(\mathrm{Pc}=0.11)$ or TaSKP_14 and TaSKP_54 genes $(\mathrm{Pc}=0.12)$. It is interesting to note that some TaSKP1-like genes had entirely opposite profiles (Pc close to -1 ) such as TaSKP_36 and TaSKP_82 $(\mathrm{Pc}=-0.96)$ or TaSKP_63 and TaSKP_65 genes $\left(\mathrm{Pc}_{\mathrm{C}}=-0.95\right)$. These results suggest that there is a strong redundancy between some TaSKP1-like genes, but also that there are strong divergences that could be related to some specialized functions (Figure 7).

(a)

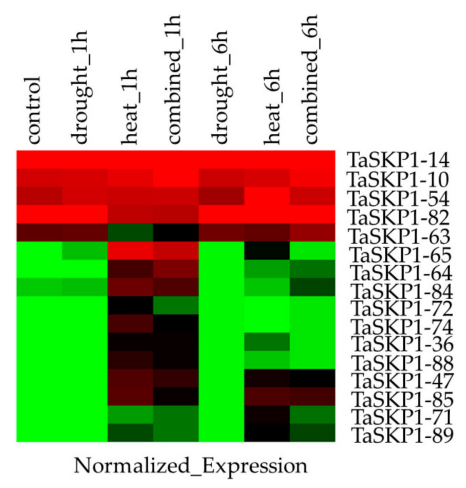

(b)

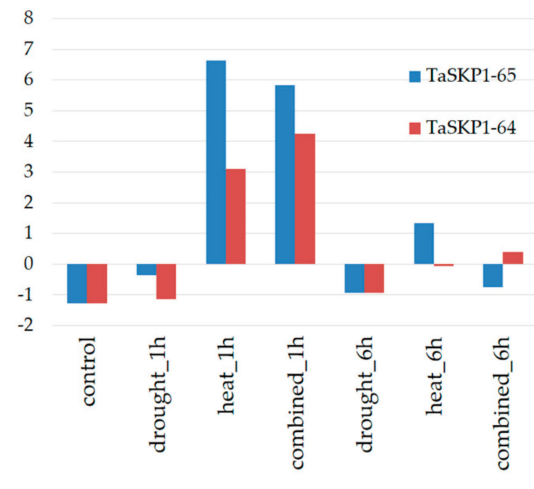

Figure 6. (a) Heatmap of 16 TaSKP1-like genes differentially expressed under $1 \mathrm{~h}$ of drought, heat and a combination of drought and heat from Liu et al. [32] and (b) expression profiles of two TaSKP1-like genes presenting a high expression under heat and a combination of heat and drought stresses.

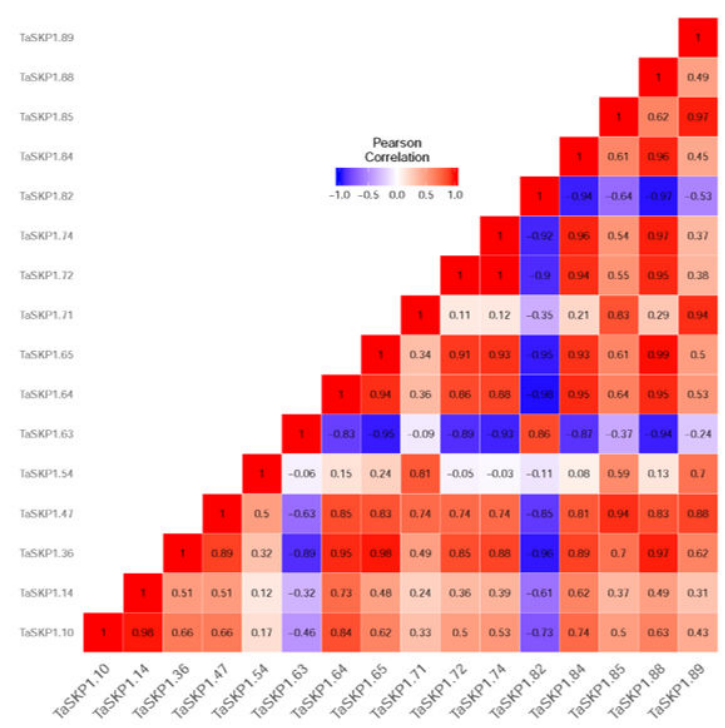

Figure 7. Pearson correlations between 16 TaSKP1 differentially expressed genes under $1 \mathrm{~h}$ of drought, heat and a combination of drought and heat from Liu et al. [32]. Dark red color indicates a strong positive correlation. Dark blue color indicates a strong negative correlation.

\subsection{Yeast Two-Hybrid (Y2H) Interaction between TaSKP1, FBX Proteins and Western Blots}

An experiment was designed to test interactions between three TaSKP1 proteins and three potential FBX interactor proteins to form SCF complexes. For each TaSKP1-FBX pair, the interaction was tested in both directions (Figure 8). 
(a)

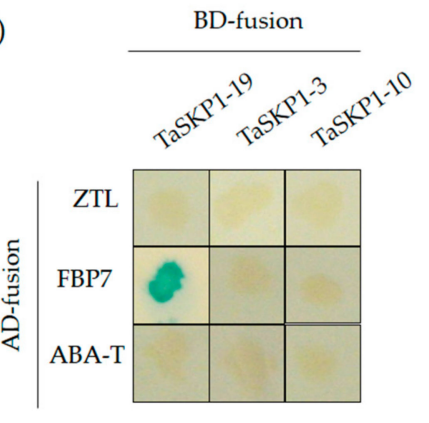

(b)

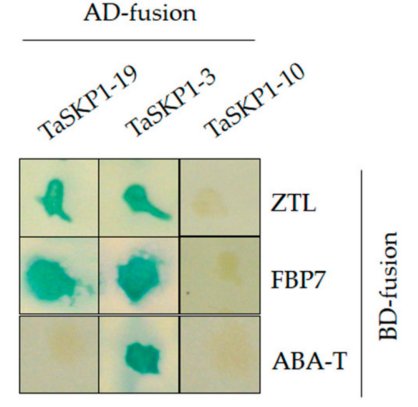

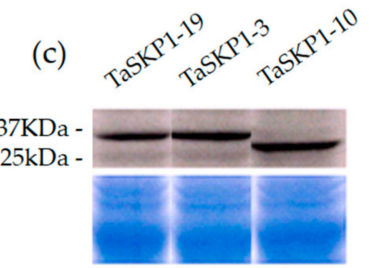

Figure 8. (a) and (b) Interactions of three TaSKP1 (TaSKP1-19, -3 and -10) proteins with three FBX proteins (ZeitLoop: ZTL, ABA-Tubby: ABA-T, and FBX7: FBP7) determined using yeast two-hybrid $(\mathrm{Y} 2 \mathrm{H})$ analysis. To generate prey (AD-fusion) and bait (BD-fusion) constructs, we fused full-length TaSKP1 and FBX proteins with the GAL4 activation and GAL4 DNA binding domains, respectively. The constructs were tested with both combinations through X-gal assays showing a blue color for positive interactions whereas non-interacting proteins remained white. (c) Immunoblot analysis of TaSKP1 and FBX proteins fused to the Gal4 DNA-binding domain.

The three TaSKP1-like genes that were selected for this study contained a single position-conserved intron. Two of these, TaSKP1_3 and TaSKP1_19 belong to a group of six TaSKP1-like genes clustered phylogenetically with six other ancestral monocot SKP1-like genes (OSK1, ZmSKP1, SbSKP1, SbSKP2, BdSKP1 and BdSKP2) (Figure 2). The third, TaSKP1_10 belongs to the second group of six TaSKP1-like genes clustered phylogenetically with three other ancestral monocot SKP1-like genes (OSK20, ZmSKP2 and SbSKP3).

TaSKP1_3 and TaSKP1_10 were moderately expressed in the four tissues analyzed (grains, spikes, leaves and roots). TaSKP1_19 was widely and highly expressed in these same tissues (Table S5).

Of the three TaSKP1 proteins examined, TaSKP1_3 was found to interact with all the FBX proteins tested (Figure 8b). TaSKP1_19 was found to interact with two of the three FBX proteins tested (Figure 8b). By contrast, TaSKP1_10 did not display any detectable interaction under our experimental conditions, even after $24 \mathrm{~h}$ of incubation at $37^{\circ} \mathrm{C}$ for X-gal assays (Figure 8a,b). In order to verify that the negative interactions were not merely due to a lack of protein production by the yeast, we examined the presence of the three TaSKP1 proteins fused to the Gal4 DNA-binding domain in yeast by western-blotting with the Gal4 monoclonal antibody. We observed accumulation of fusion proteins in TaSKP1_3, TaSKP1_10 and TaSKP1_19 (Figure 8c). Therefore, we considered that the negative interactions observed with TaSKP1_10 reflected the genuine behavior of this protein in yeast two-hybrid (Y2H) assay rather than a technical artefact due to the absence of interactors.

\section{Discussion}

\subsection{Expansion and Genomic Distribution of the TaSKP1-Like Gene Family}

The number of SKP1 genes per haploid genome varies according to the species. It ranges from one copy in humans to several dozens in certain plant species [22]. Kong et al. [26] described 31 genes in rice, 21 genes in Arabidopsis thaliana and Elzanati et al. [24] reported four genes in the moss Physcomitrella patens. In this study, we identified 92 SKP1-like genes in bread wheat (Triticum aestivum). This relatively large number could be explained partly by the hexaploid nature of wheat. Indeed, our results showed that there are 25 SKP1-like genes in subgenome A, 30 in B and 37 in subgenome D. These values are 
comparable to those found in other diploid cereals that are botanically close to wheat, such as Oryza sativa ssp. indica (31 genes) or Oryza sativa ssp. japonica (26 genes). Nevertheless, when we used the criteria suggested by the International Wheat Genome Sequencing Consortium (IWGSC) (2014) to identify homeologs (i.e., two sequences are declared as homeologs when they share at least $90 \%$ amino-acid identity over the total length of the proteins), we found eight triplets and 11 pairs yielding a total of 46 proteins. This indicates that polyploidy accounts for about half of the total number of TaSKP1-like genes, while the rest of the TaSKP1-like members have likely arisen as the result of other duplication events such as small-scale duplications and/or retroposition.

Regarding the distribution of the TaSKP1-like genes according to their structure, it appears that 40 out of the 92 genes (43\%) do not carry any intron (Class Ib). Similarly, 11 of the 24 SKP1-like genes in Fragaria vesca (46\%) and 18 of the 38 in Medicago truncatula $(47 \%)$ are also intronless. However, the proportion of this class of genes can be even higher in, for example, Oryza sativa ssp. Indica where they represented $73 \%$ (19 out of the 26 genes) and in Arabidopsis lyrata where they represented $75 \%$ (15 out of the 20 genes) [23]. Kong et al. [26] suggested that SKP1-like genes lacking introns are likely to be retroposon-mediated rearrangements. This has been demonstrated for at least one SKP1-like gene, OSK3 in rice, for which TSD (target site duplication) signatures and a polyA stretch have been found in the sequence which constitutes vestiges of a retrotransposition event. In the case of bread wheat, we scrutinized the intronless sequences for TSD and polyA signatures, but we have not been able to detect any. This is probably due to the rapid evolution of this class of genes (fast evolving genes) as suggested by Kong et al. [26] in rice and Arabidopsis [22]. The probable ancient retrotransposition accompanied by successive mutations could have modified these signatures and made them non-identifiable. Alternatively, intronless genes could result from the repair of a double strand DNA break (DSB) by non-homologous end joining (NHEJ) as was suggested by Farlow et al. [33].

In addition to the retrotransposition phenomena that contributed to the expansion of TaSKP1-like genes, other types of duplications were also probably involved. Indeed, when we compared large regions containing some TaSKP1-like genes (Figure 4), we found that they consisted of highly duplicated small regions (small-scale duplications) that occur between chromosomes of the same subgenome (for example between 4D and 7D) as well as between non-homoeologous regions (for example between $2 \mathrm{D}$ and 3B). This can be explained by the hypothesis proposed by Wicker et al. [34] according to which a double strand break (DSB) could be created upon the insertion of transposable elements (TE) and that the cellular repair machinery would use a partially homologous template from another region (ectopic recombination). If the template region contains a gene, then this gene would be duplicated. Consistent with this hypothesis, TaSKP1-like genes are located in regions enriched with repeated sequences and TEs which may favor a DSB [31]. This hypothesis was supported by Glover et al. [35] who found regions enriched with repeated sequences and TEs in the $3 \mathrm{~B}$ chromosome in bread wheat. Twenty-seven percent of filtered genes appeared to be non-syntenic, i.e., in non-conserved chromosomal location with other cereals; whereas only $8 \%$ of filtered genes seem to be non-syntenic in maize. These percentages suggest that the Triticeae lineage presents a particularly high percentage of interchromosomal duplications which is normally not a feature of large genomes. The same results in wheat were observed by Guérin et al. [36] on the expansion of the NAC (NAM (for No Apical Meristem), ATAF1 and -2 , and CUC2 (for cup-shaped cotyledon)) transcription factors.

Although we have not specifically addressed the mechanisms of gain and loss of introns in the TaSKP1-like gene family, we did identify 18 members belonging to the class of TaSKP1-like genes having a single intron but at various positions. When these genes were aligned with the nearest ancestral TaSKP1-like genes, deletions and/or insertions in the second exon, leading to its size modification were detected. For example, the TaSKP1_35 gene has undergone a deletion of 15 bases in the second exon compared to its ancestors. The same situation was found for several other TaSKP1 like genes (deletion of 39 bases in the second exon of TaSKP1_31 and TaSKP1_32, two insertions of three and six bases, i.e., nine bases in total in TaSKP_36; or deletion of three and then 46 bases, i.e., 49 bases in exon 2 of TaSKP_37 and TaSKP_38. These examples show that in addition to large genomic variations, 
short insertions/deletions can also lead to structural and probably functional diversity in this family. Schulman et al. [37] and Zheng et al. [38] have shown that the second exon contains 21 out of the 26 amino acids essential for the interaction between SKP1 and FBX proteins in humans and those amino acids are conserved in ancestral OsSKP1-like genes in rice [23]. Therefore, it is reasonable to suggest that substantial modifications affecting this second exon would be likely to significantly affect the interaction profile of these proteins with FBX proteins.

\subsection{Fate of Duplicated Genes}

Extensive studies in animal and plants have shown the prominent role of duplication in creating variations within genes which may lead to adaptive responses of species to the environment. Some of the adaptive responses could be attributed to differences in expression between the duplicated genes [39]. When we compared the expression profiles of different TaSKP1-like genes, we found that they behave differently and that they could be separated into three categories: weakly, moderately and strongly expressed genes. In addition, even if some genes are phylogenetically very similar, they can display very divergent or even opposite expression profiles. This is the case, for example, for the TaSKP_71 and TaSKP_72 genes (Pearson coefficient $=0.11$ ) or TaSKP_63 and TaSKP_65 (Pearson coefficient $=-0.95$ ). This observation is in agreement with the duplication-divergence-complementation (DDC) model proposed by Force et al. [40], which suggests that both duplicates could be maintained within a genome as a consequence of the accumulation of degenerative mutations in the regulatory regions of one duplicate leading to its subfunctionalization.

\subsection{Protein-Protein Interactions}

In addition to the analyses of the divergence in the expression profiles, we investigated the interaction abilities of some TaSKP1-like genes. It has been shown that Arabidopsis SKP1 genes possess different interaction capabilities when tested with different FBX proteins which are the direct interactors to form specific SCF E3 ligase complexes [25,41]. Similarly, it has been shown that not all SKP proteins are able to interact with all FBX in rice [23], nor in the moss Physcomitrella patens [24]; but ancestral SKP1 proteins interact with a large number of FBX proteins. A similar situation was observed in Arabidopsis thaliana with ASK1, ASK2 and ASK11 proteins [25,41]. In wheat, using the yeast two-hybrid $(\mathrm{Y} 2 \mathrm{H})$ method, the three TaSKP proteins tested were found to interact differently with the three FBX proteins tested. The TaSKP1_3 protein was able to interact with the three FBX that we tested, TaSKP1_19 with two of the FBX proteins and, finally, TaSKP1_10 did not interact with any of the FBX proteins tested. This clearly showed that these three TaSKP proteins have also diverged in their ability to interact with their FBX partners. In addition, it is interesting to note that TaSKP1_3 and TaSKP1_19 belong to an ancestral monocot group (OSK1, ZmSKP1, SbSKP1, SbSKP2, BdSKP1 and BdSKP2) (Figure 2). Therefore, we hypothesize that six TaSKP1 proteins (encoding TaSKP1_3, TaSKP1_15, TaSKP1_16, TaSKP1_19, TaSKP1_21, TaSKP1_27) are likely to have functions similar to ASK1 and ASK2 in Arabidopsis, OSK1 and OSK20 in rice and PpSKP1 and PpSKP2 in P. patens.

\section{Materials and Methods}

\subsection{Data Retrieval}

The annotated proteins in the database of the International Wheat Genome Sequencing Consortium (IWGSC RefSeq v1.0) and in the TGAC v1 [28] were searched using the HMMER2 program implemented in Unipro UGENE 1.25 [42]. As a query, the HMM (Hidden Markov Model) profile of SKP1 (PF01466) and SKP1_POZ (PF03931) domains were downloaded from Pfam. The HMM profiles were generated by alignments of 243 and 13 seed sequences (Pfam database). The HMMER2 selected proteins (with an E-value cut-off $<0.1$ ) were then scanned for SKP1 and SKP1_POZ domains using InterProScan [43] (http://www.ebi.ac.uk/interpro/search/sequence-search). The SKP1 and SKP1_POZ domains containing proteins identified by InterProScan were then used as the query sequence for 
a BLASTP search (with an E-value cut-off $<1 \times 10^{-5}$ ) of the entire wheat genome. Finally, the BLASTP hits were scanned for SKP1 and SKP1_POZ domains using InterProScan. The end results of the combination of the HMMER2, BLASTP and InterProScan searches provided the whole set of TaSKP1 proteins in the two annotated references of the wheat genome.

\subsection{Chromosomal Location of TaSKP1-Like Genes}

To map the TaSKP1-like genes on the T. aestivum L. pseudomolecule, the chromosomal coordinates of each TaSKP1-like gene were obtained from the International Wheat Genome Sequencing Consortium (https://www.wheatgenome.org/) and visualized using MapGene2Chromosome V2 (http://mg2c.iask. in/mg2c_v2.0/).

\subsection{Identification of Homoeologous Relationships between TaSKP1 Sequences}

Because bread wheat is a hexaploid species, we aimed first at identifying the homoeologous genes. For this purpose, we applied the criterion proposed by Pfeifer et al. [44] and Liu et al. [32]. The CD-HIT suite (http://weizhong-lab.ucsd.edu/cdhit-web-server/cgi-bin) was used to identify clusters of highly similar sequences with the identity cut-off set at $90 \%$ over the longest sequence. The rest of CD-HIT parameters were set as default. Highly similar sequences located on similar positions of homoeologous chromosomes were deemed homoeologs. Schematic representation of the intron-exon organization of TaSKP1-like genes was inferred using Gene Structure Display Server 2.0 (http://gsds.cbi.pku.edu.cn)

\subsection{Duplications}

When browsing the wheat pseudomolecule, we found that TaSKP1-like genes are often clustered in chromosomal areas without the presence of other genes, presumably due to duplications. In order to identify the duplication events between loci, for each locus containing a TaSKP1-like gene, $100 \mathrm{~kb}$ on either side of the gene were retrieved. In cases where multiple TaSKP1-like genes were separated by less than $100 \mathrm{~kb}$, the window of sequence retrieval was extended from the most distal SKP1 coordinates. Homologies between these sequences were then searched using Yass, a Blast-like tool [45] with an e-value threshold $<1 \times 10^{-100}$. The Yass output results were then parsed using the following criterion: sequences with at least $90 \%$ identity over at least $3 \mathrm{~kb}$ were considered duplicate. Figures showing these duplications were generated using circa software (OMGenomics, http://omgenomics.com/circa/).

\subsection{Phylogeny of the TaSKP1-Like Genes}

MEGA7 software was used to infer the unrooted phylogenetic tree, based on the alignment of the amino acid sequences of 174 SKP-like genes from moss, monocot and eudicot species comprising 92 T. aestivum TaSKP1-like genes (TaSKP1_1 through TaSKP1_92), four P. patens genes denoted PpSKP (PpSKP1 through PpSKP4), 21 A. thaliana ASK genes (ASK1 through ASK21) and 29 Oryza sativa genes (OSK1 through OSK31; two of which are no longer included in this denomination). A set of 28 additional SKP1-like genes from various plant species with a single position-conserved intron was included in the phylogeny tree. The conservation of intron position was used as a criterion to identify putative ancestral TaSKP1-like genes, as suggested by Kong et al. [26] and Kahloul et al. [23]. The position of a given intron was considered as conserved if it occurred between two aligning bases in the alignment of the coding sequences [46].

Evolutionary history was inferred using the maximum likelihood method [47]. The parameters of the constructed tree were: test of phylogeny: (a) bootstrap (500 replicates), (b) gaps/missing data treatment: partial deletion, (c) model/method LG model, (d) rates among sites: gamma distributed with invariant sites $(G)$. Only bootstrap values greater than 60 were displayed on the tree. 


\subsection{Meta-Analysis of the Expression of Wheat SKP1-Like Genes}

The expression profiles of TaSKP1-like genes were monitored in different tissues (grains, spikes, leaves and roots, data extracted from Choulet et al. [29]) or in response to abiotic stresses in leaves (heat shock and PEG-induced drought at an early stage, data extracted from Liu et al. [32]), based on the data available on the www.wheat-expression.com platform [27]. Briefly, raw expression data were downloaded and analyzed using the R Package LIMMA [48]. Weakly expressed genes were removed from the whole wheat genome expression raw data. The expression levels of the remaining genes were normalized using the TMM and Voom methods [31,48]. Genes that display an absolute log fold change $>1$ with an adjusted $p$-value $<0.05$ between two conditions were deemed Differentially Expressed Genes and noted as DEG. Hierarchical clustering of DEG was conducted using Mev software [49] with euclidean distance and average linkage.

\subsection{Plant Materials and Growth Conditions}

Recital, a winter-wheat (Triticum aestivum) variety, was used in this study. Plants were grown in soilless compost with $16 \mathrm{~h}$ light and $8 \mathrm{~h}$ darkness at $18 \pm 1{ }^{\circ} \mathrm{C}$. Light intensity was approximately $200 \mu \mathrm{E} / \mathrm{m}^{2} / \mathrm{sec}$.

\subsection{Total RNA Extraction and Isolation of TaSKP and TaFBX cDNAs}

Total RNA was isolated from two to three week old wheat plants using the method described by Bogorad et al. [50]. RNA was treated with DNaseI (Invitrogen, Carlsbad, CA, USA). First strand cDNA was synthesized using the SuperScript II reverse transcriptase and oligo-dT primer (Invitrogen). Forward and reverse primers containing AttB1 and AttB2 tails at their 5'-end (Table S6) were used to amplify TaSKP1_3, TaSKP1_10,TaSKP1_19 and three TaFBX full length cDNA (Gateway Technology, Invitrogen, Carlsbad, CA, USA). PCR products were then separated on a $1 \%$ agarose gel and purified with GFX Purification Kit (Amersham, Little Chalfont, UK).

\section{9. cDNA Cloning}

To clone genes of interest, we used $\mathrm{pENTR}^{\mathrm{TM}} / \mathrm{D}-\mathrm{TOPO} \AA$ cloning kits (Invitrogen, Carlsbad, CA, USA). PCR products were cloned directionally by adding four bases to the forward primer (CACC). All constructs were sequenced.

\subsection{Binary Yeast Two-Hybrid Analysis (Y2H)}

\subsubsection{Plasmid Constructs}

To produce hybrid proteins, Gateway Cloning Technology (Invitrogen) was used. The yeast expression vectors $\mathrm{pDEST}{ }^{\mathrm{TM}} 32$ and $\mathrm{pDEST}^{\mathrm{TM}} 22$ were used to generate GAL 4 DNA Binding Domain (GAL4BD) and GAL4 DNA Activation Domain (GAL4AD) fusion proteins. The LR reaction (Gateway Technology, Invitrogen) was performed in order to clone three TaSKP1-like genes (TaSKP1_3, TaSKP1_19 and TaSKP1_10) and three TaFBX (TaZTL-like, TaFBP7-like and TaABA-T-like) in both directions into the destination vectors.

\subsubsection{Yeast Two-Hybrid (Y2H) Screening and Assays}

Bait and prey vectors were mixed and transformed into the MaV203 yeast strain using the ProQuestTM Two-hybrid System (Invitrogen), according to the manufacturer's manual. Y2H screens and assays were performed as described previously by Kahloul et al. [23].

\subsection{Protein Extraction and Immunoblotting}

In order to verify for the real production of fusion proteins, total yeast proteins were extracted according to the method described by Printen and Sprague [51] and separated by electrophoresis with 
the Mini-PROTEAN TGX (Tris-Glycine eXtended, BioRad, Hercules, CA, USA) on either precast 10\% gels or $8 \%$ SDS-polyacrylamide gels. Immunoblot assay was performed as described previously by Kahloul et al. [23].

\section{Conclusions}

In this study, we identified 92 TaSKP1-like genes using the latest version of the wheat genome annotation. Those genes are located in regions rich in short duplications, suggesting that they have probably been expanded through small-scale duplications. Following these duplications, the TaSKP1-like genes have, in all likelihood, diverged by accumulating mutations to the point of exhibiting different expression profiles but also different interaction capabilities with their potential FBX protein interactors. Structural, phylogeny, expression and interaction data suggest that a group of six TaSKP1-like genes (TaSKP1_3, TaSKP1_15, TaSKP1_16, TaSKP1_19, TaSKP1_21, TaSKP1_27) which clustered phylogenetically with six other ancestral monocot SKP1-like genes (OSK1, ZmSKP1, $S b S K P 1, S b S K P 2, B d S K P 1$ and BdSKP2) could be proposed as functionally equivalent to ancestral SKP1 genes in plants.

Supplementary Materials: Supplementary materials can be found at http://www.mdpi.com/1422-0067/20/13/ 3295/s1.

Author Contributions: Conceptualization: I.H.E.B., S.M., M.F.B. and J.R.; formal analysis: S.M.; validation: M.-F.B. and J.R.; writing —original draft preparation: S.M.; writing—review and editing: S.M. and J.R.

Funding: This research received no external funding.

Acknowledgments: We would like to thank Patricia Mabrut for reviewing English of our manuscript and Monique Sibaud for her technical assistance. Our deep thoughts go to our dear colleague Mohammed-Fouad Bouzidi who left us the 8th of March, 2019.

Conflicts of Interest: The authors declare no conflict of interest.

\section{References}

1. Hershko, A.; Ciechanover, A. Mechanisms of intracellular protein breakdown. Annu. Rev. Biochem. 1982, 51, 335-364. [CrossRef] [PubMed]

2. Vierstra, R.D. The ubiquitin-26S proteasome system at the nexus of plant biology. Nat. Rev. Mol. Cell Biol. 2009, 10, 385-397. [CrossRef] [PubMed]

3. Genschik, P.; Marrocco, K.; Bach, L.; Noir, S.; Criqui, M.C. Selective protein degradation: A rheostat to modulate cell-cycle phase transitions. J. Exp. Bot. 2014, 65, 2603-2615. [CrossRef] [PubMed]

4. Jia, F.; Wang, C.; Huang, J.; Yang, G.; Wu, C.; Zheng, C. SCF E3 ligase PP2-B11 plays a positive role in response to salt stress in Arabidopsis. J. Exp. Bot. 2015, 66, 4683-4697. [CrossRef] [PubMed]

5. Stone, S.L.; Callis, J. Ubiquitin ligases mediate growth and development by promoting protein death. Curr. Opin. Plant Biol. 2007, 10, 624-632. [CrossRef] [PubMed]

6. Stone, S.L. The role of ubiquitin and the $26 \mathrm{~S}$ proteasome in plant abiotic stress signaling. Front. Plant Sci. 2014, 5, 135. [CrossRef] [PubMed]

7. Deshaies, R.J.; Joazeiro, C.A. RING domain E3 ubiquitin ligases. Annu. Rev. Biochem. 2009, 78, 399-434. [CrossRef] [PubMed]

8. Skaar, J.R.; Pagan, J.K.; Pagano, M. SnapShot: F-Box Proteins I. Cell 2009, 137, 1160-1161. [CrossRef]

9. Feldman, R.M.R.; Correll, C.C.; Kaplan, K.B.; Deshaies, R.J. A complex of Cdc4p, Skp1p, and Cdc53p/cullin catalyzes ubiquitination of the phosphorylated CDK inhibitor Sic1p. Cell 1997, 91, 221-230. [CrossRef]

10. Kamura, T.; Conrad, M.N.; Yan, Q.; Conaway, R.C.; Conaway, J.W. The Rbx1 subunit of SCF and VHL E3 ubiquitin ligase activates Rub1 modification of cullins Cdc53 and Cul2. Genes Dev. 1999, 13, 2928-2933. [CrossRef]

11. Gray, W.M.; del Pozo, J.C.; Walker, L.; Hobbie, L.; Risseeuw, E.; Banks, T.; Crosby, W.L.; Yang, M.; Ma, H.; Estelle, M. Identification of an SCF ubiquitin-ligase complex required for auxin response in Arabidopsis thaliana. Genes Dev. 1999, 13, 1678-1691. [CrossRef] [PubMed] 
12. Xu, L.; Liu, F.; Lechner, E.; Genschik, P.; Crosby, W.L.; Ma, H.; Peng, W.; Huang, D.; Xie, D. The SCF(COI1) ubiquitin-ligase complexes are required for jasmonate response in Arabidopsis. Plant Cell 2002, 8, 1919-1935. [CrossRef] [PubMed]

13. Nayak, S.; Santiago, F.E.; Jin, H.; Lin, D.; Schedl, T.; Kipreos, E.T. The Caenorhabditis elegans Skp1-related gene family: Diverse functions in cell proliferation, morphogenesis, and meiosis. Curr. Biol. 2002, 124, 277-287. [CrossRef]

14. Guo, H.; Ecker, J.R. Plant responses to ethylene gas are mediated by SCFEBF1/EBF2- dependent proteolysis of EIN3 transcription factor. Cell 2003, 115, 667-677. [CrossRef]

15. Ni, W.; Xie, D.; Hobbie, L.; Feng, B.; Zhao, D.; Akkara, J.; Ma, H. Regulation of Flower Development by SCF Complexes1 in Arabidopsis. Plant Physiol. 2004, 134, 1574-1585. [CrossRef]

16. Parry, G.; Estelle, M. Auxin receptors: A new role for F-box proteins. Curr. Opin. Cell Biol. 2006, 18, $152-156$. [CrossRef]

17. Porat, R.; Lu, P.; O'Neill, S.D. Arabidopsis SKP1, a homologue of a cell cycle regulator gene, is predominantly expressed in meristematic cells. Planta 1998, 204, 345-351. [CrossRef]

18. Zhao, D.; Yang, M.; Solava, J.J.; Ma, H. The ASK1 gene regulates development and interacts with the UFO gene to control floral organ identity in Arabidopsis. Dev. Genet. 1999, 25, 209-223. [CrossRef]

19. Zhao, D.; Yu, Q.; Chen, M.; Ma, H. The ASK1 gene regulates B function gene expression in cooperation with UFO and LEAFY in Arabidopsis. Development 2001, 128, 2735-2746.

20. Zhao, D.; Ni, W.; Feng, B.; Han, T.; Petrasek, M.G.; Ma, H. Members of the Arabidopsis-SKP1-like Gene Family Exhibit a Variety of Expression Patterns and May Play Diverse Roles in Arabidopsis. Plant Physiol. 2003, 133, 203-217. [CrossRef]

21. Liu, F.; Ni, W.; Griffith, M.E.; Huang, Z.; Chang, C.; Peng, W.; Ma, H.; Xie, D. The ASK1 and ASK2 Genes Are Essential for Arabidopsis Early Development. Plant Cell 2004, 16, 5-20. [CrossRef] [PubMed]

22. Kong, H.; Leebens-Mack, J.; Ni, W.; DePamphilis, C.W.; Ma, H. Highly Heterogeneous Rates of Evolution in the SKP1 Gene Family in Plants and Animals: Functional and Evolutionary Implications. Mol. Biol. Evol. 2004, 21, 117-128. [CrossRef] [PubMed]

23. Kahloul, S.; Hajsalah El Beji, I.; Boulaflous, A.; Ferchichi, A.; Kong, H.; Mouzeyar, S.; Bouzidi, M.F. Structural, expression and interaction analysis of rice SKP1-like genes. DNA Res. 2013, 20, 67-78. [CrossRef] [PubMed]

24. Elzanati, O.; Roche, J.; Boulaflous-Stevens, A.; Mouzeyar, S.; Bouzidi, M.F. Genome-wide analysis, classification, expression and interaction of Physcomitrella patens SKP1-like (PpSKP) and F-box (FBX) genes. Plant Gene 2017, 12, 13-22. [CrossRef]

25. Takahashi, N.; Kuroda, H.; Kuromori, T.; Hirayama, T.; Seki, M.; Shinozaki, K. Expression and Interaction Analysis of Arabidopsis Skp1-Related Genes. Plant Cell Physiol. 2004, 45, 83-91. [CrossRef]

26. Kong, H.; Landherr, L.L.; Frohlich, M.W.; Leebens-Mack, J.; Ma, H.; DePamphilis, C.W. Patterns of gene duplication in the plant SKP1 gene family in angiosperms: Evidence for multiple mechanisms of rapid gene birth. Plant J. 2007, 50, 873-885. [CrossRef] [PubMed]

27. Borrill, P.; Ramirez-Gonzalez, R.; Uauy, C. expVIP: A Customizable RNA-seq Data Analysis and Visualization Platform. Plant Physiol. 2016, 170, 2172-2186. [CrossRef]

28. Clavijo, B.J.; Venturini, L.; Schudoma, C.; Accinelli, G.G.; Kaithakottil, G.; Wright, J. An improved assembly and annotation of the allohexaploid wheat genome identifies complete families of agronomic genes and provides genomic evidence for chromosomal translocations. Genome Res. 2017, 27, 885-896. [CrossRef]

29. Choulet, F.; Alberti, A.; Theil, S.; Glover, N.; Barbe, V.; Daron, J.; Pingault, L.; Sourdille, P.; Couloux, A.; Paux, E.; et al. Structural and functional partitioning of bread wheat chromosome 3B. Science 2014, 345, 1249721. [CrossRef]

30. Smyth, G.K. Linear Models and Empirical Bayes Methods for Assessing Differential Expression in Microarray Experiments. Stat. Appl. Genet. Mol. Biol. 2004, 3, 1-25. [CrossRef]

31. Robinson, M.D.; Oshlack, A. A scaling normalization method for differential expression analysis of RNA-seq data. Genome Biol. 2010, 11, R25. [CrossRef] [PubMed]

32. Liu, Z.; Xin, M.; Qin, J.; Peng, H.; Ni, Z.; Yao, Y.; Sun, Q. Temporal transcriptome profiling reveals expression partitioning of homeologous genes contributing to heat and drought acclimation in wheat (Triticum aestivum L.). BMC Plant Biol. 2015, 15, 1-20. [CrossRef] [PubMed]

33. Farlow, A.; Meduri, E.; Schlöttereret, C. DNA double-strand break repair and the evolution of intron density. Trends Genet. 2011, 27, 1-6. [CrossRef] [PubMed] 
34. Wicker, T.; Buchmann, J.P.; Keller, B. Patching gaps in plant genomes results in gene movement and erosion of colinearity. Genome Res. Trends Genet. 2010, 20, 1229-1237. [CrossRef] [PubMed]

35. Glover, N.M.; Daron, J.; Pingault, L.; Vandepoele, K.; Paux, E.; Feuillet, C. Small-scale gene duplications played a major role in the recent evolution of wheat chromosome 3B. Genome Biol. 2015, 16, 188. [CrossRef] [PubMed]

36. Guérin, C.; Roche, J.; Allard, V.; Ravel, C.; Mouzeyar, S.; Bouzidi, M.F. Genome-wide analysis, expansion and expression of the NAC family under drought and heat stresses in bread wheat (T. Aestivum L.). PLoS ONE 2019, 14. [CrossRef] [PubMed]

37. Schulman, B.A.; Carrano, A.C.; Jeffrey, P.D.; Bowen, Z.; Kinnucan, E.R.E.; Finnin, M.S.; Elledge, S.J.; Harper, J.W.; Pagano, M.; Pavletich, N.P. Insights into SCF ubiquitin ligases from the structure of the Skp1-Skp2 complex. Nature 2000, 408, 381-386. [CrossRef] [PubMed]

38. Zheng, N.; Schulman, B.A.; Song, L.; Miller, J.J.; Jeffrey, P.D.; Wang, P.; Chu, C.; Koepp, D.M.; Elledge, S.J.; Paganok, M.; et al. Structure of the Cul1-Rbx1-Skp1-F boxSkp2 SCF ubiquitin ligase complex. Nature 2002, 416, 703-709. [CrossRef]

39. Panchy, N.; Lehti-Shiu, M.; Shiu, S.-H. Evolution of Gene Duplication in Plants. Plant Physiol. 2016, 171, 2294-2316. [CrossRef]

40. Force, A.; Lynch, M.; Pickett, F.B.; Amores, A.; Yan, Y.L.; Postlethwait, J. Preservation of duplicate genes by complementary, degenerative mutations. Genetics 1999, 151, 1531-1545.

41. Risseeuw, E.P.; Daskalchuk, T.E.; Banks, T.W.; Liu, E.; Cotelesage, J.; Hellmann, H.; Estelle, M.; Somers, D.E.; Crosby, W.L. Protein interaction analysis of SCF ubiquitin E3 ligase subunits from Arabidopsis. Plant J. 2003, 34, 753-767. [CrossRef] [PubMed]

42. Okonechnikov, K.; Golosova, O.; Fursov, M. UGENE team. Unipro UGENE: A unified bioinformatics toolkit. Bioinformatics 2012, 28, 1166-1167. [CrossRef] [PubMed]

43. Mulder, N.J.; Apweiler, R.; Attwood, T.K.; Bairoch, A.; Bateman, A.; Binns, D.; Bork, P.; Buillard, V.; Cerutti, L.; Copley, R.; et al. New developments in the InterPro database. Nucleic Acids Res. 2007, 35, 224-228. [CrossRef] [PubMed]

44. Pfeifer, M.; Kugler, K.G.; Sandve, S.R.; Zhan, B.; Rudi, H.; Hvidsten, T.R. International Wheat Genome Sequencing Consortium. Genome interplay in the grain transcriptome of hexaploid bread wheat. Science 2014, 345, 1250091. [CrossRef] [PubMed]

45. Noé, L.; Kucherov, G. YASS: Enhancing the sensitivity of DNA similarity search. Nucleic Acids Res. 2005, 33, 540-543. [CrossRef] [PubMed]

46. Zheng, J.; Rogozin, I.B.; Koonin, E.V.; Przytycka, T.M. Support for the Coelomata clade of animals from a rigorous analysis of the pattern of intron conservation. Mol. Biol. Evol. 2007, 24, 2583-2592. [CrossRef] [PubMed]

47. Kumar, S.; Stecher, G.; Tamura, K. MEGA7: Molecular Evolutionary Genetics Analysis Version 7.0 for Bigger Datasets. Mol. Biol. Evol. 2017, 33, 1870-1874. [CrossRef]

48. Law, C.W.; Yunshun, C.; Shi, W.; Smyth, G.K. Voom: Precision weights unlock linear model analysis tools for RNA-seq read counts. Genome Biol. 2014, 15, R29. [CrossRef]

49. Howe, E.A.; Sinha, R.; Schlauch, D.; Quackenbush, J. RNA-Seq analysis in MeV. Bioinformatics 2011, 27, 3209-3210. [CrossRef]

50. Bogorad, L.; Gubbins, E.J.; Krebbers, E.; Larrinua, I.M.; Mulligan, B.J. Cloning and physical mapping of maize plastid genes [Zea mays, corn]. Methods Enzymol. 1983, 97, 524-554.

51. Printen, J.A.; Sprague, G.F. Protein-protein interactions in the yeast pheromone response pathway: Ste5p interacts with all members of the MAP kinase cascade. Genetics 1994, 138, 609-619. [PubMed]

(C) 2019 by the authors. Licensee MDPI, Basel, Switzerland. This article is an open access article distributed under the terms and conditions of the Creative Commons Attribution (CC BY) license (http://creativecommons.org/licenses/by/4.0/). 